\title{
Identification and Cluster Analysis of Sweet Corn Based on Grain Textural Properties
}

\author{
Xiangnan Li, Guihua Lv, Jianjian Chen, Zhenxing Wu, Guojin Guo* \\ Institute of Maize and Featured Upland Crops, Zhejiang Academy of Agricultural Sciences, Dongyang, P. R. China \\ Email address: \\ 2421418604@qq.com (Xiangnan Li),lvgh@zaas.ac.cn (Guihua Lv), 181078186@qq.com (Jianjian Chen), \\ wuzhenxing.1991@163.com (Zhenxing Wu),guoguojin@yahoo.com.cn (Guojin Guo) \\ ${ }^{*}$ Corresponding author
}

\section{To cite this article:}

Xiangnan Li, Guihua Lv, Jianjian Chen, Zhenxing Wu, Guojin Guo. Identification and Cluster Analysis of Sweet Corn Based on Grain Textural Properties. Journal of Plant Sciences. Vol. 8, No. 5, 2020, pp. 177-184. doi: 10.11648/j.jps.20200805.20

Received: September 22, 2020; Accepted: October 9, 2020; Published: October 16, 2020

\begin{abstract}
The edible qualities are crucial factors for quality of Fresh-eating sweet Corn. However, the research of the edible quality at the milking stage remains largely ambiguous in sweet corn. To identify phenotypes and classify genotypes via principal component analysis and cluster analysis, the textural properties of the grain of 51 sweet corn varieties in regional tests were measured by texture analyzer. The results showed that there was high genetic variation and diversity in the grain textural properties (hardness, springiness, cohesiveness, adhesiveness, chewiness, resilience, gumminess) between the 51 sweet corn varieties. Among the variation in these textural properties, the variation in adhesiveness was the greatest, and the variation in cohesiveness was the smallest; the variation ranges were $1.145 \sim 18.190$ and $0.126 \sim 0.253$, respectively. There were very significantly positive relationships between hardness, cohesiveness, chewiness and gumminess; the correlation coefficients were greater than 0.783 . However, no significant correlation between resilience and the other traits was observed. According to principal component analysis (PCA), the above seven textural characteristics were governed by three independent principal components. The per cent contributions of the variance of the three independent principal components were $54.656 \%, 15.814 \%$ and $14.737 \%$. Hardness, springiness and resilience were the dominant factors affecting the textural properties of the sweet corn grain. According to systematic cluster analysis, the 51 sweet corn varieties could be classified into 2 groups based on their hardness values, and group 1 could be further classified into 3 subgroups based on the values of springiness and resilience. These results indicated that significant genetic differences exist in the textural properties of sweet corn grain and provided useful information for improving the edible quality of sweet corn.
\end{abstract}

Keywords: Sweet Corn, Textural Properties, Principal Component Analysis, Cluster Analysis

\section{Introduction}

Sweet corn is a subspecies of Zea mays. Compared with common corn, sweet corn carries one or more recessive gene mutations that increase the amount of polysaccharides in the endosperm [1,2]. As a recently introduced food, sweet corn is commonly consumed as fruits and vegetables, and it is harvested at the immature stage of endosperm development 20-24 days after pollination [3,4]. Sweet corn grain has a desirable taste; a unique nutrient composition; and a high-quality phyto-nutrition profile comprising water-soluble phytoglycans, sucrose, fructose, dietary fibre, vitamins, antioxidants and minerals [5]. In addition, sweet corn has advantages of a short production cycle, good market conditions and high economic benefits. With the economic development and the improvement of people's living standards, the fresh corn industry is developing rapidly. During 2011, global imports of frozen sweet corn were valued at $\$ 393$ million, and preserved sweet corn was valued at $\$ 968$ million [6]. Countries such as the US, France, Hungary and Thailand are the main export countries of sweet corn products. On the other hand, Japan, Germany, the UK, Spain, the Russian Federation and China are the main import countries of sweet corn, and the demand for sweet corn has displayed a sharp increase in the last few years. From 1998 to 2016, the global sweet corn planting area increased from 66.67 to 147 million hectares, with the total planting area increased by nearly 2.2 times in the past eighteen years $[7,8]$. 
Fresh sweet corn quality involves commercial quality, nutrient quality, end-use quality and edible quality. The commercial quality involves ear shape and size, grain size and uniformity, and pericarp colour, which can be measured by visual observations. The nutrient quality includes the composition and content of sugar, starch, proteins, amino acids, vitamins, and minerals, which can be identified by chemical analysis. The end-use quality mainly consists of grain depth, pericarp thickness, and bract colour, and the edible quality mainly involves waxiness, sweetness, hardness, fragrance and other factors that can be measured by sensory evaluation. The sensory evaluation method is subjective and influenced by cultivation practices, environmental conditions, harvest time and taste personnel preferences. Therefore, it is difficult to ensure the accuracy of the evaluation results and consistency between years [9].

A texture analyser is the main instrument that can evaluate the edible quality objectively. This type of instrument is widely used in the food industry. Its working principle involves simulated mastication through double compressions of a sample. The changes in the positions and weight of samples over time can be measured accurately, and textural properties such as hardness, springiness, adhesiveness, cohesiveness, gumminess, chewiness and resilience can be caculated [10-12]. By constantly exploring correlations between sensory evaluation and texture analysis results, researchers have developed a texture evaluation system. Zhan et al. (2007) proposed that the hardness, viscosity, adhesiveness and springiness from a texture analysis can indirectly reflect the edible quality of rice [13]. Chang et al. (2009) studied the creep properties of four varieties of cooked japonica rice by a dynamic mechanical analyser and found a positive significant relationship between the viscosity coefficient and sensory evaluation $(r=0.973)$ [14]. Chauvin et al. (2010) evaluated the relationships between compressive forces, tensile forces and sensory perception of apple and pear harvested in two different years. The texture measurements of apples $(r=0.78 \sim 0.83)$ and pears $(r=0.83)$ showed a significant correlation with sensory results for hardness, and tensile data could predict crispness of apples $(r$ $=0.88)$ and pears $(r=0.85)$ [15]. Texture analysis has the following advantages: large application range, convenient operation, and suitability for mass analysis. In this study, we measured the grain textural properties of 51 sweet corn varieties by a texture analyser, and then conducted a principal component analysis (PCA) and cluster analysis. The results of this study provide a theoretical basis for the edible quality evaluation of sweet corn.

\section{Materials and Methods}

\subsection{Plant Materials and Field Evaluation}

A total of 51 sweet corn varieties from 14 provinces were obtained from provincial seed management stations (Table 1). The varieties were planted at the Zhejiang Experimental Station of the Institute of Maize and Featured Upland Crops,
Zhejiang Academy of Agricultural Sciences, China $\left(29.16^{\circ} \mathrm{N}\right.$, $120.13^{\circ} \mathrm{E}$ ) in late March of 2020 . The soil was a clayey loam and had moderate level of fertility. The soil consisted of approximately $15.4 \mathrm{~g} / \mathrm{kg}$ organic matter, $1.66 \mathrm{~g} / \mathrm{kg}$ total nitrogen, $120.6 \mathrm{mg} / \mathrm{kg}$ available nitrogen, $7.08 \mathrm{mg} / \mathrm{kg}$ available phosphorus and $86.5 \mathrm{mg} / \mathrm{kg}$ soil available potassium. The experiment was conducted in accordance with a completely random block design. Artificial bagging pollination was carried out at the flowering stage, and the samples were harvested 21 days after pollination. Planting was performed in two rows per plot, with 15 plants per row; the plants were spaced $28 \mathrm{~cm}$ apart, and the row spacing was $67.5 \mathrm{~cm}$. The field management practices were performed according to the conventional requirements.

\subsection{Sampling Methods}

To ensure the consistency of ear growth and grain filling, the 51 sweet corn varieties were subjected to bagging treatments before the silking stage. Artificial self-pollination was then performed in the full-bloom stage. At 21 days after pollination, three ears were harvested in the early morning, and texture profile analysis (TPA) was carried out after cooking.

\subsection{Test Methods}

After removing the bracts, the sweet corn ears were cooked for $20 \mathrm{~min}$ in an electric pot. When the temperature of the sweet corn ears was $60^{\circ} \mathrm{C}$ by natural cooling, eight complete grains from the same vertical row in the middle part of the corn ear were selected for texture profile analysis (TPA). TPA was performed via a TVT-300XP texture analyser, and the parameters were as follows: the probe was a $\mathrm{P} / 36 \mathrm{R}$ device; the speed of the probe was $1 \mathrm{~mm} / \mathrm{s}$ before the test; the speed of the probe was $5 \mathrm{~mm} / \mathrm{s}$ in the test; the speed of the probe was $5 \mathrm{~mm} / \mathrm{s}$ after the test; the back embryo side of the grain faced upward, with degree of compression of $90 \%$; the interval time between two compressions was $5 \mathrm{~s}$; and the trigger force was $5 \mathrm{~g}$. The textural properties included hardness, springiness, cohesiveness, adhesiveness, chewiness, resilience and gumminess.

\subsection{Statistical Analysis}

Descriptive statistics, principal component analysis (TPA) and cluster analysis of the textural properties were performed using SPSS 13.0.

\section{Results}

\subsection{Phenotypic Analysis of Textural Properties of Sweet Corn}

The textural properties of the 51 sweet corn varieties showed a wide range of variation (Table 2). Among these textural properties, adhesiveness, chewiness, resilience and gumminess presented a coefficient of variation greater than $30 \%$. Adhesiveness showed the highest coefficient of 
variation (52.425\%) and ranged from 1.145 to 18.19035 .9 , with an average of $6.454 \pm 3.383$. Resilience and chewiness showed the next highest coefficients of variation $(39.713 \%$ and $37.849 \%$, respectively) ranged from 0.065 to 0.283 and 202.172 to 871.398 , respectively. The high coefficient of variation suggested that there were high degrees of discreteness and large genetic variations for adhesiveness, resilience and chewiness of the 51 sweet corn varieties. Cohesiveness showed the lowest coefficient of variation (14.187\%) and ranged from 0.126 to 0.253 , with an average of $0.168 \pm 0.024$, it suggested that the discrete degree of cohesiveness of the 51 sweet corn varieties was low. In addition, hardness and springiness ranged from $3715.378 \mathrm{~g}$ to $7880.488 \mathrm{~g}$ and 0.281 to 0.597 , respectively.
Phenotypic correlations were analysed between the seven textural properties, and most of them exhibited significant positive correlations with each other $(p<0.05$; Table 3 ). Highly significant positive correlations were observed between hardness, gumminess, chewiness and cohesiveness, with phenotypic correlation coefficients $\left(r_{p}\right)$ greater than 0.783 . Springiness showed a significant positive correlation with chewiness $\left(r_{p}=0.617\right)$, but there was no significant correlation observed between resilience and the other properties. These results suggest that the larger the sweet corn grain hardness and springiness are, the greater the grain chewiness increase, and the greater the sweet corn grain gumminess is, the larger the grain cohesiveness increase.

Table 1. Genotypic differences in grain textural properties among 51 sweet corn varieties.

\begin{tabular}{|c|c|c|c|c|c|}
\hline Code & Origin & Variety & Hardness & Springiness & Cohesiveness \\
\hline 1 & He Bei & Wan Tian 2015 & 6108.550 & 0.471 & 0.180 \\
\hline 2 & Bei Jing & Shuang Se Tian 1606 & 7142.643 & 0.594 & 0.183 \\
\hline 3 & Bei Jing & Si Da Tian 221 & 6368.714 & 0.579 & 0.166 \\
\hline 4 & Bei Jing & Sheng tian Ai Fei & 3893.917 & 0.454 & 0.144 \\
\hline 5 & Shan Dong & SWRB6D05 & 6539.717 & 0.594 & 0.182 \\
\hline 6 & He Nan & Zheng Tian 66 & 6424.586 & 0.363 & 0.168 \\
\hline 7 & $\mathrm{Hu}$ Bei & Tai Mei Tian Er Hao & 5699.500 & 0.500 & 0.170 \\
\hline 9 & Jiang Su & Jing Tian 14 & 5066.667 & 0.413 & 0.158 \\
\hline 10 & Shang Hai & He Tian n 3 Hao & 5171.307 & 0.518 & 0.170 \\
\hline 11 & Shang Hai & He Li 1 Hao & 5702.521 & 0.595 & 0.162 \\
\hline 12 & Shang Hai & Hu Tian 13 & 6507.371 & 0.538 & 0.177 \\
\hline 13 & Shang Hai & Hu Tian 16 & 5939.500 & 0.500 & 0.170 \\
\hline 14 & Shang Hai & Shen Ke Tian 516 & 6467.714 & 0.591 & 0.193 \\
\hline 15 & Shang Hai & Shen Ke Tian 810 & 5607.167 & 0.454 & 0.177 \\
\hline 18 & Shang Hai & Mei Guo 1 Hao & 4098.000 & 0.428 & 0.137 \\
\hline 19 & Shang Hai & Yun Tian 60 & 4916.188 & 0.444 & 0.154 \\
\hline 20 & Chong Qing & YT710 & 3793.292 & 0.404 & 0.147 \\
\hline 21 & Si Chuan & Rong Yu Tian Jiu Hao & 4505.167 & 0.389 & 0.133 \\
\hline 22 & Si Chuan & Rong Yu Tian Liu Hao & 6343.536 & 0.479 & 0.157 \\
\hline 23 & Gui Zhou & Qian Tian 201 & 5623.813 & 0.484 & 0.159 \\
\hline 24 & Zhe Jiang & Zhe Tian 11 & 4890.554 & 0.373 & 0.156 \\
\hline 25 & Zhe Jiang & Jin Yu Tian 2 Hao & 6052.667 & 0.597 & 0.154 \\
\hline 26 & Zhe Jiang & Pu Tian 1 Нао & 5750.017 & 0.444 & 0.186 \\
\hline 27 & Zhe Jiang & Sheng Tian 169 & 5710.400 & 0.281 & 0.167 \\
\hline 28 & Zhe Jiang & Sheng Tian Bai Zhu & 4864.643 & 0.488 & 0.155 \\
\hline 29 & Zhe Jiang & Shu Mei Tian 10 Hao & 5477.131 & 0.380 & 0.154 \\
\hline 30 & Zhe Jiang & Zhe Tian 20 & 6306.383 & 0.376 & 0.150 \\
\hline 31 & Zhe Jiang & Zhe Tian 358 & 4672.983 & 0.535 & 0.159 \\
\hline 32 & Zhe Jiang & Zhe Tian 67 & 3715.378 & 0.473 & 0.141 \\
\hline 35 & Zhe Jiang & Cui Tian 321 & 5780.083 & 0.576 & 0.168 \\
\hline 36 & Zhe Jiang & Jin Yin 305 & 4294.167 & 0.371 & 0.126 \\
\hline 37 & Zhe Jiang & Zhe Tai Tian 928 & 7167.333 & 0.526 & 0.253 \\
\hline 38 & Fu Jian & Hua Tai Tian 328 & 5398.844 & 0.368 & 0.174 \\
\hline 39 & Fu Jian & Yong Zhen 7 Hao & 6839.798 & 0.465 & 0.183 \\
\hline 40 & Fu Jian & Wan Xian Tian 178 & 7880.488 & 0.446 & 0.203 \\
\hline 41 & Fu Jian & Hui Tian 192 & 6623.810 & 0.523 & 0.224 \\
\hline 42 & Guang Dong & Guang Liang Tian 27 Hao & 6855.229 & 0.514 & 0.187 \\
\hline 43 & Guang Dong & E Tian 28 & 6515.500 & 0.378 & 0.172 \\
\hline 44 & Guang Dong & Jiang Tian 018 & 6299.170 & 0.453 & 0.200 \\
\hline 45 & Guang Xi & Zhong Miao Bai Tian 107 & 3854.750 & 0.331 & 0.148 \\
\hline 46 & Guang Xi & Hei Shen & 7654.000 & 0.340 & 0.200 \\
\hline 47 & Guang Xi & Gui Tian 612 & 5867.800 & 0.427 & 0.167 \\
\hline 48 & Guang Xi & Gui Tian 568 & 6329.521 & 0.478 & 0.161 \\
\hline
\end{tabular}




\begin{tabular}{|c|c|c|c|c|c|}
\hline Code & Origin & Variety & Hardness & Springiness & Cohesiveness \\
\hline 49 & Guang Xi & Gui Tian 569 & 5792.357 & 0.315 & 0.170 \\
\hline 50 & Guang Xi & Zhong Xian Tian 3 Hao & 6222.400 & 0.329 & 0.190 \\
\hline 51 & Guang Xi & Jin Mei Tian 616 & 5576.729 & 0.539 & 0.170 \\
\hline
\end{tabular}

Table 1. Continued.

\begin{tabular}{|c|c|c|c|c|c|c|}
\hline Code & Origin & Variety & Adhesiveness & Chewiness & Resilience & Gumminess \\
\hline 1 & He Bei & Wan Tian 2015 & 3.159 & 530.177 & 0.085 & 1130.974 \\
\hline 2 & Bei Jing & Shuang Se Tian 1606 & 6.493 & 783.691 & 0.136 & 1340.288 \\
\hline 3 & Bei Jing & Si Da Tian 221 & 8.469 & 632.240 & 0.088 & 1068.286 \\
\hline 4 & Bei Jing & Sheng tian Ai Fei & 4.500 & 247.675 & 0.065 & 575.521 \\
\hline 5 & Shan Dong & SWRB6D05 & 7.133 & 844.456 & 0.088 & 1231.720 \\
\hline 6 & He Nan & Zheng Tian 66 & 6.844 & 414.360 & 0.093 & 1090.562 \\
\hline 7 & $\mathrm{Hu}$ Bei & Tai Mei Tian Er Hao & 9.209 & 518.388 & 0.084 & 981.058 \\
\hline 8 & Jiang Su & Cui Tian 628 & 9.591 & 648.387 & 0.121 & 1426.879 \\
\hline 9 & Jiang Su & Jing Tian 14 & 2.556 & 352.320 & 0.078 & 824.254 \\
\hline 10 & Shang Hai & He Tian n 3 Hao & 4.031 & 453.792 & 0.094 & 901.204 \\
\hline 11 & Shang Hai & He Li 1 Hao & 14.902 & 544.740 & 0.087 & 978.220 \\
\hline 12 & Shang Hai & Hu Tian 13 & 8.094 & 750.555 & 0.283 & 1199.863 \\
\hline 13 & Shang Hai & Hu Tian 16 & 6.284 & 523.538 & 0.162 & 1020.720 \\
\hline 14 & Shang Hai & Shen Ke Tian 516 & 7.616 & 744.845 & 0.126 & 1283.040 \\
\hline 15 & Shang Hai & Shen Ke Tian 810 & 1.632 & 425.700 & 0.085 & 1050.771 \\
\hline 16 & Shang Hai & Shen Ke Tian 811 & 6.319 & 328.446 & 0.139 & 675.387 \\
\hline 17 & Shang Hai & Bai Mei Ren & 10.984 & 368.862 & 0.092 & 654.856 \\
\hline 18 & Shang Hai & Mei Guo 1 Hao & 5.965 & 230.635 & 0.244 & 565.449 \\
\hline 19 & Shang Hai & Yun Tian 60 & 4.746 & 348.393 & 0.221 & 768.793 \\
\hline 20 & Chong Qing & YT710 & 3.800 & 208.945 & 0.072 & 575.437 \\
\hline 21 & Si Chuan & Rong Yu Tian Jiu Hao & 2.234 & 248.970 & 0.076 & 620.423 \\
\hline 22 & Si Chuan & Rong Yu Tian Liu Hao & 6.808 & 486.848 & 0.108 & 1020.451 \\
\hline 23 & Gui Zhou & Qian Tian 201 & 5.669 & 447.251 & 0.157 & 898.722 \\
\hline 24 & Zhe Jiang & Zhe Tian 11 & 3.348 & 304.116 & 0.077 & 769.669 \\
\hline 25 & Zhe Jiang & Jin Yu Tian 2 Hao & 12.019 & 571.119 & 0.092 & 936.487 \\
\hline 26 & Zhe Jiang & Pu Tian 1 Hao & 1.741 & 496.224 & 0.095 & 1076.093 \\
\hline 27 & Zhe Jiang & Sheng Tian 169 & 9.147 & 279.259 & 0.100 & 961.907 \\
\hline 28 & Zhe Jiang & Sheng Tian Bai Zhu & 7.719 & 355.124 & 0.074 & 820.023 \\
\hline 29 & Zhe Jiang & Shu Mei Tian 10 Hao & 5.527 & 324.155 & 0.109 & 865.210 \\
\hline 30 & Zhe Jiang & Zhe Tian 20 & 9.142 & 375.454 & 0.072 & 951.842 \\
\hline 31 & Zhe Jiang & Zhe Tian 358 & 4.730 & 382.151 & 0.073 & 747.046 \\
\hline 32 & Zhe Jiang & Zhe Tian 67 & 4.074 & 282.583 & 0.104 & 544.827 \\
\hline 33 & Zhe Jiang & Zhe Ke Tian 6 Hao & 2.238 & 252.697 & 0.087 & 506.212 \\
\hline 34 & Zhe Jiang & Cui Tian 258 & 3.407 & 331.877 & 0.177 & 696.543 \\
\hline 35 & Zhe Jiang & Cui Tian 321 & 7.838 & 587.449 & 0.110 & 985.530 \\
\hline 36 & Zhe Jiang & Jin Yin 305 & 4.673 & 202.172 & 0.086 & 548.599 \\
\hline 37 & Zhe Jiang & Zhe Tai Tian 928 & 1.145 & 871.398 & 0.136 & 1814.431 \\
\hline 38 & Fu Jian & Hua Tai Tian 328 & 5.020 & 350.102 & 0.181 & 983.042 \\
\hline 39 & Fu Jian & Yong Zhen 7 Hao & 6.656 & 580.419 & 0.116 & 1280.172 \\
\hline 40 & Fu Jian & Wan Xian Tian 178 & 18.190 & 739.427 & 0.116 & 1635.538 \\
\hline 41 & Fu Jian & Hui Tian 192 & 3.083 & 763.709 & 0.129 & 1497.976 \\
\hline 42 & Guang Dong & Guang Liang Tian 27 Нао & 8.437 & 622.655 & 0.129 & 1316.172 \\
\hline 43 & Guang Dong & E Tian 28 & 1.546 & 424.978 & 0.094 & 1127.074 \\
\hline 44 & Guang Dong & Jiang Tian 018 & 9.412 & 562.111 & 0.101 & 1276.701 \\
\hline 45 & Guang Xi & Zhong Miao Bai Tian 107 & 6.313 & 222.261 & 0.069 & 580.906 \\
\hline 46 & Guang Xi & Hei Shen & 9.549 & 583.199 & 0.100 & 1577.265 \\
\hline 47 & Guang Xi & Gui Tian 612 & 4.143 & 425.214 & 0.108 & 979.891 \\
\hline 48 & Guang Xi & Gui Tian 568 & 9.146 & 533.337 & 0.092 & 1040.357 \\
\hline 49 & Guang Xi & Gui Tian 569 & 10.299 & 319.581 & 0.087 & 1021.100 \\
\hline 50 & Guang Xi & Zhong Xian Tian 3 Hao & 7.274 & 404.308 & 0.103 & 1215.999 \\
\hline 51 & Guang Xi & Jin Mei Tian 616 & 7.713 & 513.930 & 0.098 & 965.882 \\
\hline
\end{tabular}


Table 2. Statistical analysis of textural properties of the grain of 51 sweet corn varieties.

\begin{tabular}{lllllll}
\hline Trait & Mean & Max. & Min. & Range & SD & CV (\%) \\
\hline Hardness & 5666.314 & 7880.488 & 3715.378 & 4165.110 & 1034.024 & 18.249 \\
Springiness & 0.461 & 0.597 & 0.281 & 0.316 & 0.082 & 17.822 \\
Cohesiveness & 0.168 & 0.253 & 0.126 & 0.127 & 0.024 & 14.187 \\
Adhesiveness & 6.454 & 18.190 & 1.145 & 17.045 & 3.383 & 52.425 \\
Chewiness & 463.352 & 871.398 & 202.172 & 669.226 & 175.375 & 37.849 \\
Resilience & 0.113 & 0.283 & 0.065 & 0.218 & 0.045 & 39.713 \\
Gumminess & 992.085 & 1814.431 & 506.212 & 1308.219 & 300.306 & 30.270 \\
\hline
\end{tabular}

SD Standard deviation, CV (\%) Coefficient of variation (\%)

Table 3. Correlation analysis of textural properties of 51 sweet corn varieties.

\begin{tabular}{llllllll}
\hline Trait & Hardness & Springiness & Cohesiveness & Adhesiveness & Chewiness & Resilience & Gumminess \\
\hline Hardness & 1.000 & & & & & & \\
Springiness & 0.176 & 1.000 & & & & \\
Cohesiveness & $0.783^{* *}$ & 0.154 & 1.000 & & & \\
Adhesiveness & $0.388^{* *}$ & 0.178 & 0.110 & 1.000 & & \\
Chewiness & $0.824^{* *}$ & $0.617^{* *}$ & $0.787^{* *}$ & $0.303^{*}$ & 1.000 & 1.000 & \\
Resilience & 0.113 & 0.107 & 0.102 & -0.025 & 0.175 & 0.118 \\
Gumminess & $0.942^{* *}$ & 0.176 & $0.939^{* *}$ & $0.283^{*}$ & $0.857^{* *}$ & 1.000 \\
\hline
\end{tabular}

* Significant at $P<0.05, * *$ Significant at $P<0.01$

\subsection{Principal Component Analysis of Grain Textural Properties of Sweet Corn}

Principal component analysis (PCA) is a kind of statistical method that is used to reduce multidimensional data to fewer dimensions while retaining most of the information [16]. PCA was performed to study the grain textural properties of sweet corn. The eigenvalues and contributions of the principal components are shown in Table 4 . There are three principal components (PCs) with eigenvalues greater than 1.0, and their eigenvalues are 3.826, 1.107 and 1.032. The cumulative contribution rate of the first three principal components to the total variance is $85.206 \%$, which means that the first three principal components represent more than $80 \%$ of the information of all grain textural properties of the sweet corn.

After the standardization of the initial data, the principal component scores of the first three principal components could be obtained (Table 5). The first principal component (PC. 1) could be calculated with the formula $\mathrm{Y} 1=$
$0.384-X 10.166 \times 2+0.323 \times 3-0.031 \times 4+0.165 \times 5-0.002 \times 6+0$. $319 \times 7$, it explained $54.656 \%$ of the total variance. The representative variables of the first principal component included hardness, cohesiveness and gumminess, which displayed significantly positive correlations with each other. The first principal component mostly reflected the grain hardness of sweet corn during taste. The second principal component (PC. 2) could be calculated with the formula Y2 $=-0.062 \mathrm{X} 1+0.735 \mathrm{X} 2-0.201 \mathrm{X} 3+0.373 \mathrm{X} 4+0.365 \mathrm{X} 5+0.129 \mathrm{X} 6-$ $0.131 \times 7$ and represented $15.814 \%$ of the total variance; the representative variables were springiness, adhesiveness and chewiness. The second principal component mostly reflected the grain springiness of sweet corn. The formula of the third principal component (PC. 3) is $\mathrm{Y} 3$ $=-0.095 \mathrm{X} 1+0.107 \mathrm{X} 2+0.080 \mathrm{X} 3-0.555 \mathrm{X} 4+0.065 \mathrm{X} 5+0.791 \mathrm{X} 6-$ $0.014 \times 7$. The third principal component mainly represented the grain resilience of sweet corn and explained $14.737 \%$ of the total variance. These results suggested that the hardness, springiness and resilience were important textural properties for evaluating the edible quality of sweet corn.

Table 4. Eigenvalues and contribution percentages of the principal components.

\begin{tabular}{llll}
\hline Principal components & Eigenvalue & Contribution percentage of the variance (\%) & Cumulative contribution percentage of the variance (\%) \\
\hline 1 & 3.826 & 54.656 & 54.656 \\
2 & 1.107 & 15.814 & 70.469 \\
3 & 1.032 & 14.737 & 85.206 \\
4 & 0.836 & 11.941 & 97.147 \\
5 & 0.167 & 2.386 & 99.533 \\
6 & 0.028 & 0.401 & 99.934 \\
7 & 0.005 & 0.066 & 100.000 \\
\hline
\end{tabular}

Table 5. Principal component scores after standardization.

\begin{tabular}{llll}
\hline Trait & PC. 1 & PC. 2 & PC. 3 \\
\hline Hardness & 0.384 & -0.062 & -0.095 \\
Springiness & -0.166 & 0.735 & 0.107 \\
Cohesiveness & 0.323 & -0.201 & 0.080 \\
Adhesiveness & -0.031 & 0.373 & -0.555 \\
Chewiness & 0.165 & 0.365 & 0.065 \\
\hline
\end{tabular}




\begin{tabular}{llll}
\hline Trait & PC. 1 & PC. 2 & PC. 3 \\
\hline Resilience & -0.002 & 0.129 & 0.791 \\
Gumminess & 0.319 & -0.131 & -0.014 \\
\hline
\end{tabular}

\subsection{Cluster Analysis of Grain Textural Properties of Sweet Corn}

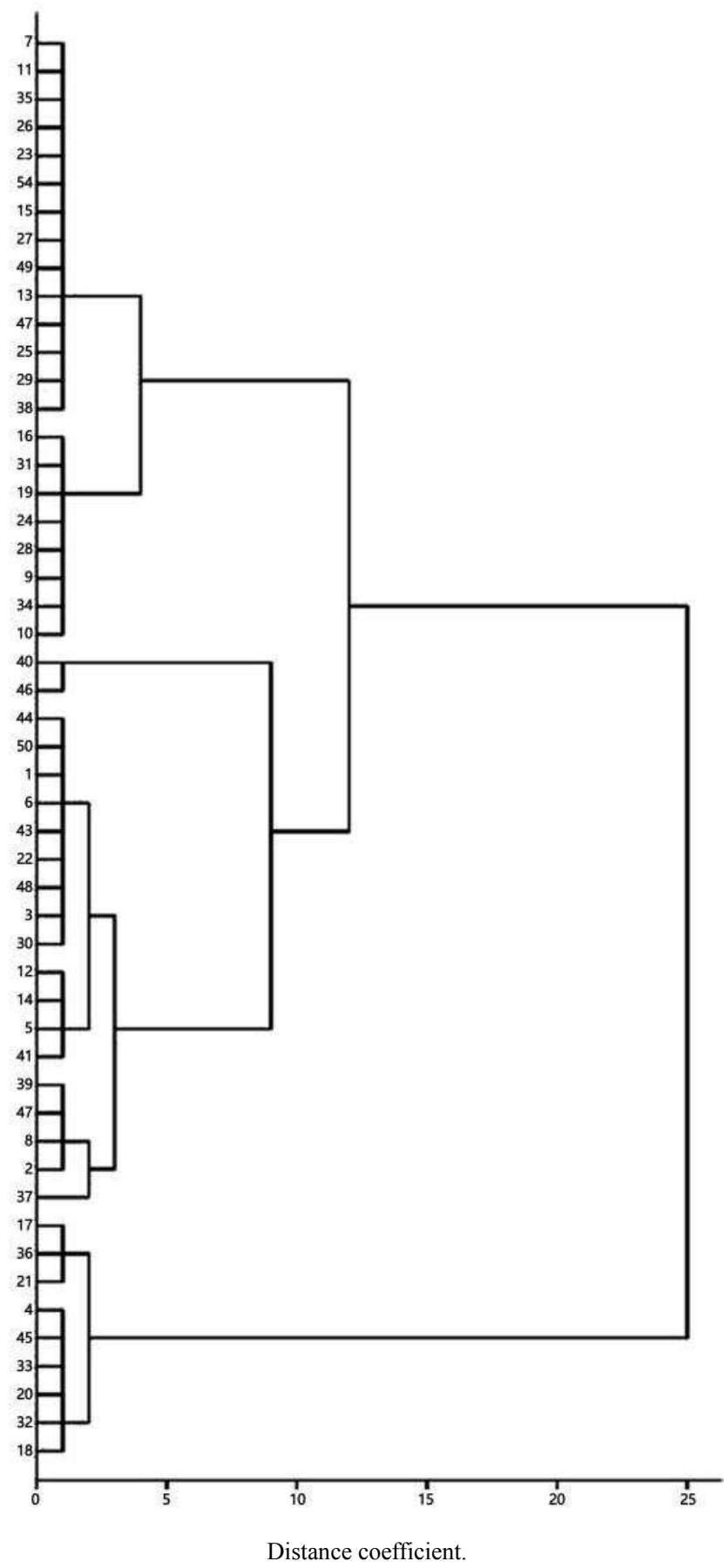

Figure 1. Clustering analysis of 51 sweet corn varieties based on textural properties.

Cluster analysis is a nonparametric statistical method that classifies groups based on the degree of similarity of samples. On the basis of PCA, cluster analysis is carried out by using the unweighted pair group method of arithmetic mean
(UPGMA). The similarity of the varieties was displayed by the euclidean distances between them (Figure 1). According to the key variable hardness of PC. 1, the 51 sweet corn varieties could be divided into two groups with a euclidean distance of 25 . Group 1 includes forty-four varieties with a high average hardness of approximately $6021.903 \mathrm{~g}$. Group 2 contained only seven varieties, whose average hardness was $4036.617 \mathrm{~g}$. At a euclidean distance of 8.84, group 1 could be further classified into three subgroups on the basis of springiness and resilience. Subgroup 1 contained twenty-two varieties: 9 varieties from Zhejiang, 6 varieties from Shanghai, 3 varieties from Guangxi, 1 variety from Jiangsu, 1 variety from Fujian, 1 variety from Hubei, and 1 variety from Guangzhou. All the varieties of subgroup 1 had high hardness, medium springiness and medium resilience. Subgroup 2 included only two varieties: Wan Xian Tian 178 from Fujian and Hei Shen from Guangxi. These varieties have characteristics that include high hardness, low springiness and resilience. Subgroup 3 comprised twenty varieties that mainly originated from provinces such as Guangdong, Zhejiang, Guangxi, and Fujian. All the varieties of subgroup 3 had high hardness, high springiness and high resilience.

\section{Discussion}

A texture analyser is a kind of instrument that can quickly, scientifically and objectively evaluate the textural properties of food. A large number of studies have confirmed that a texture analyser-based evaluation is a good supplement and substitute for sensory evaluations [17, 18]. Texture analysers have been applied extensively in the agriculture and food industries $[19,20]$. However, there are few studies on the textural properties of sweet corn. Sun et al. determined the optimum test conditions of the textural properties of ready-to-eat corn with a texture analyser and established a prediction model of sensory evaluation that could explain $69.7 \% \sim 98.7 \%$ of the total variation [21]. Lu et al calculated the TPA comprehensive evaluation (D) values of seven sweet corn varieties at different harvest times; there was a significant difference between sweet corn varieties, and an increasing $\mathrm{D}$ value could be observed with the delay of harvest time [22]. Significant differences in textural properties were also observed between 91 waxy corn varieties. For these textural properties, the $\mathrm{CV}$ of adhesiveness was the highest, and the $\mathrm{CV}$ of cohesiveness was the lowest [23]. In this study, the CV of adhesiveness was $52.425 \%$, and the CV of cohesiveness was $14.187 \%$, which was consistent with the results of previous studies.

The quality of fresh corn mainly involves the edible quality, commercial quality, end-use quality and nutrition quality, among which the edible quality is the key of the quality evaluation standard of fresh corn. The application of texture analysers in the evaluation of edible quality is very 
important for the quality improvement of fresh corn. In a previous study, 40 sweet corn varieties were investigated via texture analysis, and the variation ranges of hardness, springiness and resilience were 5075.86 9847.38 g, $0.28 \sim 0.47$ and $0.13 \sim 0.23$, respectively, their average values were $7175.67 \mathrm{~g}, 0.37$ and 0.18 [24]. In our study, the variation ranges of hardness, springiness and resilience were $3715.378 \sim 7880.488 \mathrm{~g}, \quad 0.281 \sim 0.597$ and $0.065 \sim 0.283$, respectively; the average values were $5666.314 \mathrm{~g}, 0.461$ and 0.113 . It suggested that there was greater genetic variation for springiness and resilience of the 51 sweet corn varieties, compared with the previous study, and many new sweet corn varieties which had low hardness, high springiness and low resilience could be found in this study. These results were consistent with the improving targets of low-hardness breeding in sweet corn.

\section{Conclusion}

In this study, there were significant positive correlations between the majority of textural properties. The phenotypic correlation coefficient $(r p)$ ranged from 0.283 to 0.942 . According to PCA, there were three principal components (PCs) with eigenvalues greater than 1.0. The cumulative contribution percentage of the first three principal components to the total variance was $85.206 \%$. Hardness, springiness and resilience were important textural properties for evaluating the edible quality of sweet corn. Via cluster analysis, 51 sweet corn varieties were classified into 2 groups according to the values of hardness, and group 1 was further classified into 3 subgroups based on the values of springiness and resilience. The results of this study provide useful information for improving the edible quality of sweet corn.

\section{Acknowledgements}

This work was supported by the Training Program of Young Talents in Zhejiang Academy of Agricultural Sciences (10102000320CC3001G/003/033), the Public Welfare and Technology Application Reseach Project of Zhejiang Province (LGN20C020001), and the Supporting Disciplines in Zhejiang Academy of Agricultural Sciences.

\section{References}

[1] James M, Robertson D, Myers A. Characterization of the maize gene sugaryl, a determinant of starch composition in kernels. Plant Cell. 1995; 7: 417-429.

[2] Feng Z, Liu J, Fu F, Li W. Molecular mechanism of sweet and waxy in maize. Intl J Plant Breed Genet. 2008; 2: 93-100.

[3] Khanduri A, Prasanna B, Hossain F, Lakhera P. Genetic analysis and association studies of yield components and kernel sugar concentration in sweet corn. Indian J Genet. 2010; 70: 257-263.

[4] Khanduri A, Hossain F, Lakhera P, Prasanna B. Effect of harvest time on kernel sugar concentration in sweet corn. Indian J Genet. 2011; 71: 231-234.
[5] Tracy W, Shuler S, Dodson-Swenson H. Sweet corn [M]// The physiology of vegetable crops. 2020.

[6] FAOSTAT. 2014. http://faostat.fao.org.

[7] Liang Q, Yin Y. The cultivation technology of sweet corn. Yun Nan Agriculture. 2002; 000 (003): 9-11. (in Chinese).

[8] Ni Y. Study on the development of sweet corn industrialization in Huizhou. Zhongkai University of Agriculture and Engineering. 2018. (in Chinese).

[9] Liu P, Wang C, Wang F, et al. Changes of quality components in waxy corn kernels after pollination. Scientia Agricultural Sinica. 2007; 40 (8): 1817-1821. (in Chinese).

[10] Lin F, Jiang Z, Liao S. Texture analyzer and its application in food quality evaluation. Life Sciences and Scientific Instruments. 2009; (5): 61-63. (in Chinese).

[11] Kuang F, Liu Q, Cao Q, Feng Y. Application of texture analyzer in food industries. Food Science and Technology. 2020; (03): 112-115. (in Chinese).

[12] Bourne M. Food texture and viscosity. New York: Academic Press, 2002: 254-262.

[13] Zhan X, Zheng T, Tao J. Study on application of texture analyzer in quality evaluation of Rice. Journal of Food Science. 2007; 28 (9): 62-65. (in Chinese).

[14] Chang s, Li D, Lan Y, Ozkan N. Study on creep properties of japonica cooked rice and its relationship with rice chemical compositions and sensory evaluation. International Journal of Food Engineering. 2009; 5 (3): 1-16.

[15] Chauvin M, Ross C, Pitts M, Kupferman E, Swanson B. Relationship between instrumental and sensory determination of apple and pear texture. Journal of Food Quality. 2010; 33 (2): 181-198.

[16] Karamizadeh S, Abdullah S, Manaf A, Zamani M, Hooman A. An overview of principal component analysis. Journal of Signal and Information Processing. 2013; 4 (3B): 173-175.

[17] Zhang Y, Liu J, Zhou X, et al. Study on the correlation between instrument evaluation and sensory evaluation of rice palatabilit. Journal of grain processing. 2015; 040 (003): 2630. (in Chinese).

[18] Hu Y. Application Situation of Texture Analyzer in the Study of Food. Food Research and Development. 2013; 000 (011): 101-104. (in Chinese).

[19] Farcuh M, Copes B, Le-Navenec G, et al. Texture diversity in melon (Cucumis melo L.): Sensory and physical assessments. Postharvest Biology and Technology. 2020; 159: 111024.

[20] Flores D, Giovanni M, Kirk L, et al. Capturing and explaining sensory differences among organically grown vegetable-soybean varieties grown in Northern California: Capturing and explaining sensory difference. Journal of Food Science, 2019; 84 (3): 613-622.

[21] Sun H. Study on rheological properties evaluation system of instant corn. Jilin Agricultural University. 2011. (in Chinese).

[22] Lu B, Dong H, Xu L, et al. Relationship between water content and physical properties and quality of sweet corn at different harvesting periods. Acta Agriculturae Boreali-sinica. 2019; 34 (S1): 69-77. (in Chinese). 
[23] Lu D, Wang X, Yan B, et al. Genotypic difference and principal component analysis for grain textural properties of fresh waxy Maize. Journal of Food Science. 2013; (21): 16-19. (in Chinese).
[24] Zhao F, Jing L, Yan B, et al. Genotypic difference in textural properties of sweet corn grain. Jiangsu Journal of Agricultural Science. 2013; 29 (1): 14-19. 